PROCEEDINGS OF THE

AMERICAN MATHEMATICAL SOCIETY

Volume 139, Number 4, April 2011, Pages 1321-1331

S 0002-9939(2010)10570-1

Article electronically published on September 3, 2010

\title{
HECKE ALGEBRAS RELATED TO THE UNIMODULAR AND MODULAR GROUPS OVER QUADRATIC FIELD EXTENSIONS AND QUATERNION ALGEBRAS
}

\author{
MARTIN RAUM \\ (Communicated by Wen-Ching Winnie Li)
}

\begin{abstract}
We investigate the structure of the Hecke algebras related to the unimodular and modular groups over quadratic field extensions and quaternion algebras. In particular, we show that in general there is no decomposition into primary components. We give a set of generators, and in some special cases we deduce the commutation relation with the Siegel $\Phi$-operator.
\end{abstract}

\section{INTRODUCTION}

Much arithmetic information of modular forms is related to the action of the associated Hecke algebras, i.e. the attached $\zeta$-functions. Moreover, the study of $\zeta$-functions attached to a quaternion algebra itself reveals interesting properties. This case has been investigated in Tam63. It is closely related to the unimodular quaternion case that we consider here.

The notion of an abstract Hecke algebra was first introduced by Shimura [Shi59]. Since then Hecke algebras relevant for modular forms have been investigated for many groups defined over principal ideal domains Kri90, Kri87. By using the Satake isomorphism, very important rationality results And69, HS83 can be deduced. These results hold for local Hecke algebras. The global picture could only be built in the case of groups over principal ideal domains. The case of non principal ideal domains remains mysterious until now. In Ens08 evidence has been provided that it behaves differently. We are going to discuss this example in view of this paper in Section 5. In particular, we will show how to construct double cosets, which do not split as a product of $p$-components, in an associated graded algebra.

We prove that the Hecke algebras in question for hermitian fields and definite quaternion algebras are subalgebras of their adelic analogs. The point of view suggested by this discovery makes it possible to deduce a set of generators and to determine how far the algebras are from having a primary decomposition.

In addition, in the PID case we are able to deduce the yet unproven commutation relation with the Siegel $\Phi$-operator.

The main tool for these investigations is the elementary divisor theory presented in [Rau10. There the author proved that the equivalence class of any unimodular

Received by the editors December 22, 2009 and, in revised form, April 27, 2010 and May 2, 2010.

2010 Mathematics Subject Classification. Primary 11F60.

(C)2010 American Mathematical Society Reverts to public domain 28 years from publication 
or modular matrix in quadratic extensions of fields and in quaternion algebras is completely determined by its adelic elementary divisors.

The paper is organized as follows. In Section 2 we prove that the integral Hecke algebra is a subalgebra of the adelic one. In Section 3 we determine the structure of the adelic Hecke algebra. Both results are combined in Section 4 to investigate the structure of the integral Hecke algebra. In particular, in subsection 4.1 we consider the Siegel $\Phi$-operator over $\mathbb{Q}$. Finally, in Section 5 we present examples illustrating the results.

\section{Embeddings of integral Hecke algebras}

Let $K$ be a number field and let $\mathfrak{o}_{K}$ denote its ring of integers. Depending on the case we consider, $\Omega$ will either denote a field extension of $K$ or a central simple $K$-algebra and $\Lambda$ will always be a maximal order of $\Omega$. Note that we do not exclude $K=\Omega$. The set of finite places of $\Omega$ will be denoted by $\Omega_{\mathbb{A}}^{f}$, and the set of all infinite places $\Omega_{\mathbb{A}}^{\infty}$. For $\mathfrak{p} \in \Omega_{\mathbb{A}}^{f}$ the completions of $\Omega, \Lambda$, etc., with respect to $\mathfrak{p}$ will be denoted by $\Omega_{\mathfrak{p}}, \Lambda_{\mathfrak{p}}$, etc. The $\Omega_{\mathbb{A}}^{\infty}$-integral adelic algebras associated to $\Omega$ and $\Lambda$ will be denoted by $\Omega_{\mathbb{A}}$ and $\Lambda_{\mathbb{A}}$.

Let $n \in \mathbb{N}$, and whenever $\Lambda$ is noncommutative assume $n \geq 2$. For a finite place $\mathfrak{p} \in \Omega_{\mathbb{A}}^{f}$ let $\widetilde{G L}_{n}\left(\Lambda_{\mathfrak{p}}\right)$ be the group generated by all matrices $I_{n}+a I_{i, j}^{(n, n)}$ where $i \neq j$ and $a \in \Lambda_{\mathfrak{p}}$. Here the identity matrix is denoted by $I_{n}$ and the $n \times n$ matrix with only zeros but a single one in the $i$-th row and $j$-th column is denoted by $I_{i, j}^{(n, n)}$.

The general linear group $\mathrm{GL}_{n}\left(\Lambda_{\mathfrak{p}}\right)$ is generated by $\widetilde{\mathrm{GL}}_{n}\left(\Lambda_{\mathfrak{p}}\right)$ and the diagonal matrices. This holds because $\Lambda_{\mathfrak{p}}$ is a matrix algebra over a division algebra which is a left and a right PID (cf. Rei75).

Define $\widetilde{\mathrm{GL}}_{n}\left(\Lambda_{\mathbb{A}}\right) \subseteq \mathrm{GL}_{n}\left(\Lambda_{\mathbb{A}}\right)$ to be the group of all matrices $M$ such that $M_{\mathfrak{p}} \in \widetilde{\mathrm{GL}}_{n}\left(\Lambda_{\mathfrak{p}}\right)$ for all finite places $\mathfrak{p}$ of $\Lambda$. Moreover, we will consider the monoid of adelic invertible matrices $\operatorname{Inv}_{n}\left(\Lambda_{\mathbb{A}}\right)=\mathrm{GL}_{n}\left(\Omega_{\mathbb{A}}\right) \cap \mathrm{M}_{n}\left(\Lambda_{\mathbb{A}}\right)$. In analogy, define $\widetilde{\mathrm{GL}}_{n}\left(\Lambda_{\mathfrak{p}}\right), \operatorname{Inv}_{n}\left(\Lambda_{\mathfrak{p}}\right)$, etc. (cf. Rau10).

Two elements $a, b \in \Lambda$ are called equivalent, denoted by $a \sim b$, if there are units $u, v \in \Lambda^{\times}$such that $a=u b v$. The notion of equivalence extends to $\Lambda_{\mathfrak{p}}$ and $\Lambda_{\mathbb{A}}$.

If there is an involution $\imath$ of the algebra extension $\Omega \mid K$ which fixes $K$ elementwise and preserves maximal orders, we can define the set of all matrices with similitude $m \in K^{\times}$. In the case of quadratic fields this will be an involution of the second kind; in the case of a quaternion algebra it will be of the first kind according to Siegel (cf. [Sie51]).

We denote the transpose matrix by $M^{\text {tr }}$ and define $M^{*}:=\imath(M)^{\operatorname{tr}}$ for any matrix $M \in \mathrm{M}_{n}(\Omega)$ :

$$
\Delta_{n}(\Omega, m):=\left\{M \in \mathrm{M}_{2 n}(\Omega): M^{*} J M=m J\right\} \quad \text { with } \quad J=\left(\begin{array}{cc}
0^{(n, n)} & I_{n} \\
-I_{n} & 0^{(n, n)}
\end{array}\right) .
$$

The monoid $\Delta_{n}(\Omega):=\bigcup_{m \in \mathfrak{o}_{K} \backslash\{0\}} \Delta_{n}(\Omega, m)$ of all matrices with integral similitude contains the modular group

$$
\operatorname{Sp}_{n}(\Omega):=\bigcup_{m \in \mathfrak{o}_{K}^{\times}} \Delta_{n}(\Omega, m)
$$

These notions can be extended to $\Lambda_{\mathbb{A}}$ and all subrings of $\Lambda_{\mathbb{A}}$ which are stable under $\imath$. 
We point out that most authors considering the modular group, in particular Siegel, use $m=1$ instead of $m \in \mathfrak{o}_{K}^{\times}$. We have to use this slightly extended notion here since there is no longer any canonical choice of similitudes for $\Delta_{n}$.

Recall the definition of a Hecke algebra. Let $G$ be a group and let $G \subseteq H$ be a monoid such that each double coset $G h G, h \in H$, considered as a set is a finite union of mutually distinct right cosets $G h_{i}, h_{i} \in H$. Then the Hecke algebra $\mathcal{H}(G, H)$ over $\mathbb{Z}$ is defined to be the free $\mathbb{Z}$-module with basis $\{G h G: h \in H\}$. To define the multiplication of two double cosets $G h G$ and $G \tilde{h} G$, fix one system of representatives $h_{i}$ and $\tilde{h}_{j}$ for their right coset decompositions. Recall that a multiset is a set $A$ and a map $A \rightarrow \mathbb{N}$ representing the multiplicity of any $a \in A$. We will denote them by $\left[a_{1}, \ldots\right]$. There is a well defined multiset of double cosets $\left[G \hat{h}_{i} G\right]_{i}$ such that $\left[G h_{i} \tilde{h}_{j}\right]_{i, j}=\bigcup_{i} \bigcup_{j}\left[G \hat{h}_{i, j}\right]$, where $G \hat{h}_{i} G=\biguplus_{j} G \hat{h}_{i, j}$. Hence the product $G h G \cdot G \tilde{h} G=\sum_{i} G \hat{h}_{i} G$ is well defined.

We will call the Hecke algebras associated to groups which have coefficients in $\Lambda$ the integral Hecke algebras. The terms local Hecke algebra and adelic Hecke algebra will be used analogously.

We will treat several settings in parallel. In the unimodular case we assume $\Omega$ to be a finite central simple $K$ algebra. In the modular case we distinguish the hermitian and the quaternionic cases. We assume that $\Omega \mid K$ is either a quadratic extension of fields and $\imath$ is the Galois conjugation or that $\Omega$ is a quaternion algebra and $\imath$ is its canonical involution, which restricts itself to the Galois conjugation on every maximal subfield of $\Omega$ (cf. Rei75]).

We first point out that the approximation lemmata given in Rau10 can be used to show that the right coset decomposition of any double coset and its adelic decomposition into the right coset are in one-to-one correspondence.

Lemma 2.1. Let $M \in \operatorname{Inv}_{n}(\Lambda)$ and $\operatorname{GL}_{n}\left(\Lambda_{\mathbb{A}}\right) M \mathrm{GL}_{n}\left(\Lambda_{\mathbb{A}}\right)=\biguplus_{i} \operatorname{GL}_{n}\left(\Lambda_{\mathbb{A}}\right) M_{i}$ with $\tilde{M}_{i} \in \operatorname{Inv}_{n}\left(\Lambda_{\mathbb{A}}\right)$. Then there are integral matrices $\hat{M}_{i} \in \operatorname{Inv}_{n}(\Lambda)$ such that we have $\mathrm{GL}_{n}\left(\Lambda_{\mathbb{A}}\right) M_{i}=\mathrm{GL}_{n}\left(\Lambda_{\mathbb{A}}\right) \hat{M}_{i}$ and $\mathrm{GL}_{n}(\Lambda) M \mathrm{GL}_{n}(\Lambda)=\biguplus_{i} \mathrm{GL}_{n}(\Lambda) \hat{M}_{i}$.

In analogy, given $m \in \mathfrak{o}_{K} \backslash\{0\}, M \in \Delta_{n}(\Lambda, m)$ we can find $M_{i} \in \Delta_{n}(\Lambda, m)$ such that $\operatorname{Sp}_{n}\left(\Lambda_{\mathbb{A}}\right) M \operatorname{Sp}_{n}\left(\Lambda_{\mathbb{A}}\right)=\biguplus_{i} \operatorname{Sp}_{n}\left(\Lambda_{\mathbb{A}}\right) M_{i}$ and $\operatorname{Sp}_{n}(\Lambda) M \operatorname{Sp}_{n}(\Lambda)=\biguplus_{i} \operatorname{Sp}_{n}(\Lambda) M_{i}$.

We only prove the unimodular case, since the modular case is completely analogous.

Suppose that there is an adelic right coset $\mathrm{GL}_{n}\left(\Lambda_{\mathbb{A}}\right) M^{\prime} \subseteq \mathrm{GL}_{n}\left(\Lambda_{\mathbb{A}}\right) M \mathrm{GL}_{n}\left(\Lambda_{\mathbb{A}}\right)$ with $M^{\prime} \in \operatorname{Inv}_{n}\left(\Lambda_{\mathbb{A}}\right)$. Then there is $V \in \widetilde{G L}_{n}\left(\Lambda_{\mathbb{A}}\right)$ such that the right cosets statisfy $\mathrm{GL}_{n}\left(\Lambda_{\mathbb{A}}\right) M V=\mathrm{GL}_{n}\left(\Lambda_{\mathbb{A}}\right) M^{\prime}$. Choose $m \in \mathfrak{o}_{K} \backslash\{0\}$ such that we have $m M^{-1}, m M^{\prime-1} \in \mathrm{M}_{n}\left(\Lambda_{\mathbb{A}}\right)$. Also choose an approximation $V \in \mathrm{GL}_{n}(\Lambda)$ modulo $m \Lambda_{\mathbb{A}}$ of $V$. Then $M \hat{V} M^{\prime-1}$ and $M^{\prime}(M \hat{V})^{-1}$ are integral in $\mathrm{M}_{n}\left(\Omega_{\mathbb{A}}\right)$, and hence $\mathrm{GL}_{n}\left(\Lambda_{\mathbb{A}}\right) M \hat{V}=\mathrm{GL}_{n}\left(\Lambda_{\mathbb{A}}\right) M^{\prime}$.

On the other hand a standard argument shows that two right cosets which are distinct with respect to $\Lambda$ are also distinct with respect to $\Lambda_{\mathbb{A}}$.

Remark 2.2. As pointed out in Rau10 this proof is constructive. Namely, given an adelic double coset we can determine a set of integral right coset representatives.

The lemma immediately yields a structure theorem for integral Hecke algebras.

Theorem 2.3. The integral unimodular Hecke algebra $\mathcal{H}\left(\mathrm{GL}_{n}(\Lambda), \operatorname{Inv}_{n}(\Lambda)\right)$ and the modular Hecke algebra $\mathcal{H}\left(\operatorname{Sp}_{n}(\Lambda), \Delta_{n}(\Lambda)\right)$ are subalgebras of the adelic analogs $\mathcal{H}\left(\operatorname{GL}_{n}\left(\Lambda_{\mathbb{A}}\right), \operatorname{Inv}_{n}\left(\Lambda_{\mathbb{A}}\right)\right)$ and $\mathcal{H}\left(\operatorname{Sp}_{n}\left(\Lambda_{\mathbb{A}}\right), \Delta_{n}\left(\Lambda_{\mathbb{A}}\right)\right)$, respectively. The embeddings 
map a double coset over $\Lambda$ generated by $M$ to the adelic double coset generated by $M$.

Suppose $\# \mathrm{Cl}\left(\mathfrak{o}_{K}\right)=1$. Then the embedding yields an isomorphism if and only if $\Lambda$ is a principal ideal domain.

Proof. We only treat the modular case. By Lemma 2.1 there is an inclusion of the underlying module $\mathbb{Z}\left[\operatorname{Sp}_{n}(\Lambda) M, M \in \Delta_{n}(\Lambda)\right]^{\mathrm{Sp}_{n}(\Lambda)}$ into the underlying module $\mathbb{Z}\left[\operatorname{Sp}_{n}\left(\Lambda_{\mathbb{A}}\right) M, M \in \Delta_{n}\left(\Lambda_{\mathbb{A}}\right)\right]^{\operatorname{Sp}_{n}\left(\Lambda_{\mathbb{A}}\right)}$. Since multiplication is defined by means of multiplication of double cosets, this extends to an embedding of algebras.

If $\Lambda$ is a PID the inclusion of $\mathbb{Z}$-modules is obviously trivial. Suppose that $\# \mathrm{Cl}(\Lambda) \neq 1$. Then by $\left[\operatorname{Rau10}\right.$, Theorem 3] there is a double coset in $\Delta_{n}\left(\Lambda_{\mathbb{A}}\right)$ which is disjoint from $\Delta_{n}(\Lambda)$. This shows that the inclusion is proper.

We want to point out that the exceptional modular case $\Omega=K$ and $\imath=\operatorname{id}_{K}$, which corresponds to the symplectic group, builds up the complete picture. Here the integral and the adelic Hecke algebra are isomorphic.

The adelic Hecke algebra inherits a tensor product decomposition from $\Lambda_{\mathbb{A}}$. So does the Hecke algebra over $\Lambda$ whenever it is a principal ideal domain. We will see that this is no longer true for the Hecke algebra over an arbitrary $\Lambda$ which is not a principal ideal domain. The first evidence for this behavior in the hermitian modular case was found in Ens08 by means of elementary methods. This phenomenon will be investigated in Section 4 .

\section{Adelic Hecke algebras}

The first question arising from the preceding section is how we can understand the structure of the adelic Hecke algebras. This is easy for the unimodular case. We immediately see that

$$
\mathcal{H}\left(\operatorname{GL}_{n}\left(\Lambda_{\mathbb{A}}\right), \operatorname{Inv}_{n}\left(\Lambda_{\mathbb{A}}\right)\right) \cong \bigotimes_{\mathfrak{p} \in \Omega_{\mathbb{A}}^{f}} \mathcal{H}\left(\operatorname{GL}_{n}\left(\Lambda_{\mathfrak{p}}\right), \operatorname{Inv}_{n}\left(\Lambda_{\mathfrak{p}}\right)\right)
$$

The modular case is more involved. Surprisingly, the quaternionic case is much easier, since the canonical automorphism acts trivially on $\Omega_{\mathbb{A}}^{f}$. This yields a decomposition of the adelic Hecke algebra analogous to the unimodular case:

$$
\mathcal{H}\left(\operatorname{Sp}_{n}\left(\Lambda_{\mathbb{A}}\right), \Delta_{n}\left(\Lambda_{\mathbb{A}}\right)\right) \cong \bigotimes_{\mathfrak{p} \in \Omega_{\mathbb{A}}^{f}} \mathcal{H}\left(\operatorname{Sp}_{n}\left(\Lambda_{\mathfrak{p}}\right), \operatorname{Inv}_{n}\left(\Lambda_{\mathfrak{p}}\right)\right)
$$

One important case remains to be treated. This is the split hermitian modular case. The Galois conjugation acts nontrivially on split prime ideals in $\Lambda$. For the associated $\mathfrak{p} \in \Omega_{\mathbb{A}}^{f}$ define the monoid of restricted invertible matrices $\operatorname{Inv}_{n}^{\text {Res }}\left(\Lambda_{\mathfrak{p}}\right) \subseteq \operatorname{Inv}_{n}\left(\Lambda_{\mathfrak{p}}\right) \times \mathbb{N}_{0}$ to be the set of all pairs $(M, l)$ such that $\mathfrak{p}^{l} M^{-1}$ is integral.

Let us first present the result:

$$
\begin{aligned}
& \mathcal{H}\left(\operatorname{Sp}_{n}\left(\Lambda_{\mathbb{A}}\right), \Delta_{n}\left(\Lambda_{\mathbb{A}}\right)\right) \\
\cong & \bigotimes_{\mathfrak{p}=\imath(\mathfrak{p}) \in \Omega_{\mathbb{A}}^{f}} \mathcal{H}\left(\operatorname{Sp}_{n}\left(\Lambda_{\mathfrak{p}}\right), \Delta_{n}\left(\Lambda_{\mathfrak{p}}\right)\right) \otimes \bigotimes_{\{\mathfrak{p}, \imath(\mathfrak{p})\}: \mathfrak{p} \neq \imath(\mathfrak{p}) \in \Omega_{\mathbb{A}}^{f}} \mathcal{H}\left(\operatorname{Sp}_{n}\left(\Lambda_{\mathfrak{p}} \oplus \Lambda_{\overline{\mathfrak{p}}}\right), \Delta_{n}\left(\Lambda_{\mathfrak{p}} \oplus \Lambda_{\overline{\mathfrak{p}}}\right)\right) \\
\cong & \bigotimes_{\mathfrak{p}=\imath(\mathfrak{p}) \in \Omega_{\mathbb{A}}^{f}} \mathcal{H}\left(\operatorname{Sp}_{n}\left(\Lambda_{\mathfrak{p}}\right), \Delta_{n}\left(\Lambda_{\mathfrak{p}}\right)\right) \otimes \bigotimes_{\{\mathfrak{p}, \imath(\mathfrak{p})\}: \mathfrak{p} \neq \imath(\mathfrak{p}) \in \Omega_{\mathbb{A}}^{f}} \bigotimes^{\mathcal{H}}\left(\operatorname{GL}_{n}\left(\Lambda_{\mathfrak{p}}\right), \operatorname{Inv}_{n}^{\operatorname{Res}}\left(\Lambda_{\mathfrak{p}}\right)\right) .
\end{aligned}
$$


Here $\operatorname{GL}_{n}\left(\Lambda_{\mathfrak{p}}\right)$ acts trivially on the second component of $\operatorname{Inv}_{n}^{\operatorname{Res}}\left(\Lambda_{\mathfrak{p}}\right)$. The first isomorphism is obvious since the definition of modular matrices only involves $M$ and $\bar{M}$.

We have to prove that

$$
\mathcal{H}\left(\operatorname{Sp}_{n}\left(\Lambda_{\mathfrak{p}} \oplus \Lambda_{\overline{\mathfrak{p}}}\right), \Delta_{n}\left(\Lambda_{\mathfrak{p}} \oplus \Lambda_{\overline{\mathfrak{p}}}\right)\right) \cong \mathcal{H}\left(\mathrm{GL}_{n}\left(\Lambda_{\mathfrak{p}}\right), \operatorname{Inv}_{n}^{\operatorname{Res}}\left(\Lambda_{\mathfrak{p}}\right)\right)
$$

Write $\left(M_{\mathfrak{p}}, M_{\imath(\mathfrak{p})}\right)=M \in \Delta_{n}\left(\Lambda_{\mathfrak{p}} \oplus \Lambda_{\overline{\mathfrak{p}}}, m\right)$, where $m \in \operatorname{Fix}_{\imath}\left(\Lambda_{\mathfrak{p}} \oplus \Lambda_{\overline{\mathfrak{p}}}\right) \backslash\{(0,0)\}$. The restriction $M^{*} J M=m J$ is equivalent to $M_{\imath(\mathfrak{p})}=m J^{-1}\left(M_{\mathfrak{p}}^{-1}\right)^{*} J$. Since $M_{\imath(\mathfrak{p})}$ has to be integral, $m M_{\mathfrak{p}}^{-1}$ has to be integral too, and this completely determines $M$. The right and double coset decompositions only depend on the first component, and this proves the statement.

The theorems on generators of primary components given in Kri90 and Kri87] generalize to all local Hecke algebras mentioned above. To prove this we can use the normal p-rank. For any double coset we will call the rank of any representative over $\Lambda / \mathfrak{p} \Lambda$ its $\mathfrak{p}$-rank.

Let $\left[B_{1}, \ldots, B_{i}\right], i \in \mathbb{N}$, denote a block diagonal matrix. We have $\Lambda_{\mathfrak{p}} \cong \mathrm{M}_{r}(D)$ for some $r \in \mathbb{N}$ and some local division algebra $D$. We will denote a prime element of $D$ by $\pi_{D}$.

Lemma 3.1. (1) A set of generators of $\mathcal{H}\left(\mathrm{GL}_{n}\left(\Lambda_{\mathfrak{p}}\right), \operatorname{Inv}_{n}\left(\Lambda_{\mathfrak{p}}\right)\right)$ is given by the double cosets $T_{i}$ for $i=1, \ldots, r n$. Each $T_{i}$ is the double coset associated to $\left[I_{n r-i}, \pi_{D} I_{i}\right] \in \mathrm{M}_{n r}(D) \cong \mathrm{M}_{n}\left(\Lambda_{\mathfrak{p}}\right)$.

(2) In the inert hermitian modular case suppose $\pi_{D}=\overline{\pi_{D}}$. A set of generators of $\mathcal{H}\left(\operatorname{Sp}_{n}\left(\Lambda_{\mathfrak{p}}\right), \Delta_{n}\left(\Lambda_{\mathfrak{p}}\right)\right)$ is given by $T_{i}$ with $i=0, \ldots$, rn. Here $T_{0}$ is the double coset associated to $\left[I_{n r}, \pi_{D} I_{n r}\right]$. The $T_{i}, i=1, \ldots, r n$, are double cosets associated to $\left[I_{r n-i}, \pi_{D} I_{i}, \pi_{D}^{2} I_{r n-i}, \pi_{D} I_{i}\right]$.

(3) In the ramified quaternionic case and in the ramified hermitian modular case a set of generators of $\mathcal{H}\left(\operatorname{Sp}_{n}\left(\Lambda_{\mathfrak{p}}\right), \Delta_{n}\left(\Lambda_{\mathfrak{p}}\right)\right)$ is given by double cosets $T_{i}$ with $i=0, \ldots, r n$. The $T_{i}, i=0, \ldots, r n$, are double cosets associated to $\left[I_{r n-i}, \pi_{D} I_{i}, \pi_{D} \overline{\pi_{D}} I_{r n-i}, \pi_{D} I_{i}\right]$.

(4) In the split hermitian modular case a set of generators of the Hecke algebra $\mathcal{H}\left(\mathrm{GL}_{2 n}\left(\Lambda_{\mathfrak{p}}\right), \operatorname{Inv}_{2 n}^{\operatorname{Res}}\left(\Lambda_{\mathfrak{p}}\right)\right)$ is given by $T_{i}, i=0, \ldots, 2 n$. The $T_{i}$ are double cosets generated by $\left[I_{2 n-i}, \pi_{D} I_{i}\right]$. The second component of each $T_{i}$ is 1 .

(5) In the split quaternionic modular case a set of generators of the Hecke algebra $\mathcal{H}\left(\operatorname{Sp}_{n}\left(\Lambda_{\mathfrak{p}}\right), \Delta_{n}\left(\Lambda_{\mathfrak{p}}\right)\right)$ is given by $S, S^{\prime}$ and $R_{2}, \ldots, R_{2 n}$. Here $S$ is generated by $\left[I_{2 n}, \pi_{D} I_{2 n}\right]$ and $S^{\prime}$ is generated by $\left[I_{2 n-1}, \pi_{D}, \pi_{D} I_{2 n-2}, 1, \pi_{D}\right]$. The $R_{2 i}$ are generated by $\left[I_{2 n-2 i}, \pi_{D} I_{2 i}, \pi_{D}^{2} I_{2 n-2 i}, \pi_{D} I_{2 i}\right]$, and the $R_{2 i-1}$ are generated by $\left[I_{2 n-2 i+1}, \pi_{D} I_{2 i-1}, \pi_{D}^{2} I_{2 n-2 i}, \pi_{D}, \pi_{D}^{2}, \pi_{D} I_{2 i-2}\right]$.

All these Hecke algebras are commutative, and all sets of generators are algebraically independent.

Remark 3.2. In (1) notice that the condition on the elementary divisors in $D$ corresponds to the condition $e_{i} \| e_{i+1}$ imposed on the generators over the Hurwitz order given in [Kri87.

Proof. These statements are essentially contained in Krieg's work, which we cited above. We first proof the statements on generators.

Let us first consider the unimodular case. We have $\Lambda_{\mathfrak{p}}=\mathrm{M}_{r}(D)$ for some local division algebra $D$. Hence, we can assume that $\Lambda_{\mathfrak{p}}$ is a division algebra. Given some nontrivial double coset $M$ choose $1 \leq i \leq n$ such that $\hat{e}_{n-i}(M) \neq 0$ and 
$\hat{e}_{n-i-1}(M)=0$. Let $M^{\prime}$ be the double coset with $\hat{e}_{j}\left(M^{\prime}\right)=\hat{e}_{j}(M)$ if $j<n-i$ and $\hat{e}_{j}\left(M^{\prime}\right)=\hat{e}_{j}(M)-1 \geq 0$ otherwise. Using the p-rank as in Kri90, Theorem V.8.2] we can prove that $T_{i} M^{\prime}=M+\sum_{i} R_{i}$, where all $R_{i}$ have $\mathfrak{p}$-rank less than $n-i$. Using induction on the p-rank proves statement (11).

In the symplectic case we can reduce the split field extension case to the unimodular case. In all other cases we can apply the ideas given above. More precisely, in the quaternion case the proofs can be done along the lines of [Kri87, Theorem 3.8]. There, Krieg essentially exploits the local structure of the Hurwitz order. Notice that 2 is the only place ramified in this quaternion algebra. The hermitian case is the local field case. Hence, we treat the modular group over a PID. The results follow from Kri90, Theorem VI.6.2].

In all cases but the split hermitian modular case, the last statements follow from the existence of the canonical anti isomorphism of the Hecke algebra given by $M \mapsto \imath(M)^{\operatorname{tr}}$. By the elementary divisor theory given in [Rau10, this is indeed the identity. The commutativity in the split hermitian modular case follows from the unimodular case.

The algebraic independence of the generators follows from counting the double cosets with fixed maximal elementary divisors or fixed similitude, respectively, as Krieg has done in Kri87.

To investigate the structure and determine generators of the integral Hecke algebra we need to prove one further lemma. It is a vast generalization of well known lemmata on the multiplication of certain elements of the primary components.

For the rest of this section we will only be concerned with the local Hecke algebras. We will identify double cosets $M$ with the ordered set of their defining elementary divisor valuations $\hat{e}(M):=\left(\hat{e}_{1}(M) \leq \cdots \leq \hat{e}_{r n}(M)\right)$ in the unimodular case. In the modular case if $\mathfrak{p}$ is ramified we set $\left(\hat{e}(M):=\left(\hat{e}_{1}(M) \leq \cdots \leq \hat{e}_{r n}(M)\right), l\right)$, where $m \sim \mathfrak{p}^{l}$ and $2 \mid l$. The split hermitian modular case is an exception. In this case we will identify the double cosets with $\left(\hat{e}(M):=\left(\hat{e}_{1}(M) \leq \cdots \leq \hat{e}_{2 n}(M)\right), l\right)$ where $m \sim(\mathfrak{p} \imath(\mathfrak{p}))^{l}$. Here $\hat{e}_{i}(M)$ is the valuation of the $i$-th elementary divisor $e_{i}(M)$.

We introduce an order on the modular double cosets:

$$
\begin{aligned}
(\hat{e}(M), l)<\left(\hat{e}\left(M^{\prime}\right), l^{\prime}\right) & : \Leftrightarrow\left(l<l^{\prime}\right) \vee\left(l=l^{\prime} \wedge \hat{e}(M)<\hat{e}\left(M^{\prime}\right)\right) \\
\hat{e}(M)<\hat{e}\left(M^{\prime}\right) & : \Leftrightarrow \exists i: \hat{e}_{i}(M)>\hat{e}_{i}\left(M^{\prime}\right) \wedge \forall j<i: \hat{e}_{j}(M)=\hat{e}_{j}\left(M^{\prime}\right) .
\end{aligned}
$$

The order on unimodular cosets is given as above, ignoring the similitude valuation $l$. Notice that in the second line the ordering symbols are reversed. We use this notation to obtain a usual filtration on the Hecke algebra (cf. Remark 3.4).

The important fact about the order given above is that it induces a very useful filtration on the Hecke algebra. This will lead to a better understanding of products of arbitrary double cosets.

Lemma 3.3. Given double cosets $M$ and $N$ with respect to the symplectic group, we have $M N=P+\sum_{j} R_{j}$, where $\hat{e}(P)=\hat{e}(M)+\hat{e}(N)$ and $R_{j}$ are double cosets satisfying $R_{j}<P$.

Remark 3.4. This shows that the order given above induces a filtration on the Hecke algebra. We will exploit the structure of the associated graded ring in the next section. 
Proof. To unburden notation we consider the unimodular case. The symplectic case differs only by the similitude, which does not change the argument.

We prove that if $M<N$, the leading term of $T_{i} M$ is smaller than the leading term of $T_{i} N$ for all generators $T_{i}$ given above. We will use induction on $\hat{e}(M)$ with respect to the order given above. Using the $\mathfrak{p}$-rank we can write $M=S M^{\prime}+\sum_{j} R_{j}$ with a generator $S$ and $R_{j}<M$ for all $j$. We apply the induction hypothesis to the product of $S$ and the analogous decomposition of $M^{\prime}$. Iterating this, $M$ can be written in terms of generators and the rest $\sum_{j} R_{j}^{\prime}$, where $R_{j}^{\prime}<M$.

We can write $T_{i} M=T_{i} \prod_{j} S_{j}+\sum_{j} T_{i} R_{j}$. Reordering the generators $S_{j}$ and $T_{i}$ we see that the leading term $M^{\prime}$ of the product satisfies $\hat{e}\left(M^{\prime}\right)=\hat{e}(M)+\hat{e}\left(T_{i}\right)$. This proves our intermediate statement.

Now, the final statement can be proved by repetition of the argument. Namely, we first decompose $M$ and $N$ into a product of generators up to the rest that is strictly less. Then we calculate the product of these generators, which gives the leading term and the rest as stated above.

\section{The integral Hecke Algebra}

In Section 2 we have already shown that the integral Hecke algebras are subalgebras of the adelic ones. We will now provide a set of generators and prove some results about it that make their computation possible. One special case which gave rise to the presented investigation will be given explicitly. Here we can even prove the minimality of a set of generators with respect to inclusion.

In this section all $\hat{e}$ and $\hat{e}_{i}$ will be indexed by finite places of $\Lambda$. In particular, let $\hat{e}(M)$ denote the tuple of all local elementary divisor valuations $\left(\hat{e}_{\mathfrak{p}}(M)\right)_{\mathfrak{p} \in \Omega_{\mathbb{A}}^{f} \text {. Let }}$ $G$ be the set of all double cosets $M$ in $\operatorname{Inv}_{n}(\Lambda)$ or $\Delta_{n}(\Lambda)$ such that whenever the elementary divisor valuations satisfy $\hat{e}(M)=\hat{e}\left(N_{1}\right)+\hat{e}\left(N_{2}\right)$ for two double cosets $N_{1}, N_{2}$ over $\Lambda$, it follows that one of them is trivial. We will call the double cosets in $G$ irreducible. This is motivated by the fact that they are irreducible in the graded algebra associated to the integral Hecke algebra with rational coefficients according to Lemma 3.3. Notice that in [Ens08] Ensenbach called some double cosets satisfying this property indecomposable.

Using the definition of $G$ and Lemma 3.3 we immediately see that $G$ generates the Hecke algebra as a $\mathbb{Z}$-algebra. This makes the irreducible double coset important, and we want to deduce an upper bound for the number of places with nontrivial local elementary divisors as well as for the number of distinct local elementary divisors.

We may regard any local elementary divisor as an element of the Picard group. In Rau10] we proved that an adelic double coset is the image of an integral double coset if and only if the sum of its elementary divisors vanishes in $\operatorname{Pic}(\Lambda)$. We denote the order of the Picard group by $h_{\Lambda}$ and its exponent by $e_{\Lambda}$. Furthermore, we consider the fundamental divisor valuations $\hat{e}$ of a matrix or double coset. We set $\hat{f}_{i}:=\hat{e}_{i}-\hat{e}_{i-1}$ with $\hat{e}_{0}:=(0)_{\mathfrak{p} \in \Omega_{\AA}^{f}}$. These are the fundamental divisor valuations.

Suppose $h_{\Lambda} \neq 1$. Any double coset with $\hat{f}_{\mathfrak{p}, i} \geq e_{\Lambda}$ for some $\mathfrak{p}$ and $i$ is decomposable. Consider a double coset $M$ and assume $\hat{f}_{\mathfrak{p}, i}<e_{\Lambda}$ for all indices. Suppose that there are at least $b_{\Lambda}:=\left(h_{\Lambda}-1\right)\left(e_{\Lambda}-1\right)+1$ pairwise distinct and nonconjugated places $\mathfrak{p} \in \Omega_{\mathbb{A}}^{f}$ such that $\hat{f}_{\mathfrak{p}}(M)$ is not trivial. Then we may find a subset of $e_{\Lambda}$ 
places $\mathfrak{p}_{j}$ and indices $i_{j}$ such that in $\operatorname{Pic}(\Lambda)$ we have $\sum_{j} \mathfrak{p}^{\eta_{j}} \cdot\left(n+1-i_{j}\right)=0$ with exponents $\eta_{j}=\hat{f}_{\mathfrak{p}_{j}, i_{j}}$. Hence $M$ is decomposable.

This proves

Lemma 4.1. The set $G$ of irreducible double cosets generate the Hecke algebra. Moreover, there is an upper bound for the number of places where any irreducible double coset is nontrivial, and there is an upper bound for the valuation of the local elementary divisors.

Since the irreducibility of any double coset depends only on the valuation of its elementary divisors and the images of the local left ideals in the Picard group, there are indeed only finitely many cases we have to consider. In the quaternion modular case the Picard group's exponent divides 2, and hence the set of irreducible double cosets is easy to derive. In the hermitian modular case the set of irreducible double cosets may become rather large. Using the following idea the computation is feasible.

First consider a decomposition into cyclic $p$-groups $\operatorname{Pic}(\Lambda) \cong \bigoplus_{i=1}^{r}\left(C_{p_{i}^{\eta_{i}}}\right)^{l_{i}}$ with pairwise distinct pairs $\left(p_{i}, \eta_{i}\right)$. Notice that any integral double coset vanishes in each component of the Picard group. A double coset is irreducible if and only if it is irreducible with respect to each of the $p$-components $\bigoplus_{i: p_{i}=p}\left(C_{p_{i}^{\eta_{i}}}\right)^{l_{i}}$. Hence, by applying a basis transformation, we may assume that $\operatorname{Pic}(\Lambda) \cong C_{p^{\eta}}$ for one prime $p$ and some $\eta \in \mathbb{N}$.

Consider the multisets $\left[c_{j}\right]_{j}$ of elements in $\operatorname{Pic}(\Lambda)$ such that $\sum_{i} \tilde{c}_{i}=0$. Then for any submultiset $\left[\tilde{c}_{i}\right]_{i}$ we have $\sum_{i} c_{i} \neq 0$. Given one of these multisets consider the multisets of pairs of places and indices $\left(\mathfrak{p}_{j}, i_{j}\right)$ satisfying $\mathfrak{p}_{j}\left(n+1-i_{j}\right)=c_{i}$. These multisets are in a one-to-one correspondence to irreducible double cosets $M$ by virtue of

$$
\forall \mathfrak{p} \forall i \in\{1, \ldots, n\}: \#\left\{j: \mathfrak{p}_{j}=\mathfrak{p} \vee i_{j}=i\right\}=\hat{f}_{\mathfrak{p}, i}(M) .
$$

According to Lemma 4.1 we only have to consider multisets of length at most $\left(p^{\eta}-1\right)^{3}$. So transversing all of them we can list all representatives of irreducible double cosets. To get the desired double cosets over some ideal $\mathfrak{n}$ of $\Lambda$ it suffices to determine the image of all prime divisors in the Picard group.

4.1. The commutation relation with the Siegel $\Phi$-operator. The application of these results to the theory of modular forms renders the following question important. One is interested in proving that the Siegel $\Phi$-operator is surjective. Suppose that $K=\mathbb{Q}$ and that $\Lambda$ is a principal ideal domain. To simplify the notation we let $\tilde{N}(\delta)=\delta$ for given $\delta \in \Omega$ if $\Omega$ is commutative, and $\tilde{N}(\delta)=\delta \imath(\delta)$ otherwise. Notice that $\tilde{N} \imath(\tilde{N})$ equals $\#\left(\Lambda_{\mathfrak{p}} / \mathfrak{p} \Lambda_{\mathfrak{p}}\right)$ (cf. [Rei75]).

Using this notation suppose that $k \in \mathbb{N}$ such that $k$ divides the exponent of $N\left(\Lambda^{\times}\right)$. In the field extension case we have to assume that $\Lambda$ is hermitian, since otherwise $N\left(\Lambda^{\times}\right)$will not be finite. We also suppose that $n \geq 2$, if $\Lambda$ is commutative, and $n \geq 3$ otherwise. We consider the underlying modules of linear combinations of right cosets as mentioned in Section 2 to define the map

$$
\Phi_{k}: \mathbb{Q} \otimes \mathcal{H}\left(\operatorname{Sp}_{n}(\Lambda), \Delta_{n}(\Lambda)\right) \rightarrow \mathbb{Q} \otimes \mathcal{H}\left(\operatorname{Sp}_{n-1}(\Lambda), \Delta_{n-1}(\Lambda)\right) .
$$


Fix a set of right coset representatives $h_{i}$ of a double coset $M$ and suppose

$$
h_{i}=\left(\begin{array}{cccc}
A_{i} & 0^{(n-1,1)} & B_{i} & * \\
* & \alpha_{i} & * & * \\
C_{i} & 0^{(n-1,1)} & D_{i} & * \\
0^{(1, n-1)} & 0 & 0^{(1, n-1)} & \delta_{i}
\end{array}\right) .
$$

Then we define $\Phi_{k}(M)=\sum_{i} \tilde{N}\left(\delta_{i}\right)^{-k} \operatorname{Sp}_{n}(\Lambda)\left(\begin{array}{cc}A_{i} & B_{i} \\ C_{i} & D_{i}\end{array}\right) \operatorname{Sp}_{n}(\Lambda)$.

Since we identified the integral Hecke algebra with the adelic one, our final theorem can be proved by a standard counting argument.

Theorem 4.2. Let $\Omega \mid \mathbb{Q}$ be a hermitian field or a quaternion algebra with class number $\# \mathrm{Cl}(\Omega)=1$. Let $k \in \mathbb{N}$ such that $k$ divides the exponent of $N\left(\Lambda^{\times}\right) \in\{1,2\}$. If $\Omega$ is hermitian, suppose $n \geq 2$; otherwise $n \geq 3$.

The map $\Phi_{k}$ is surjective except if $\Lambda$ is noncommutative and $k=2 n-1$ or $k=2 n-2$.

Remark 4.3. Notice that if $\Lambda$ is not a principal ideal domain, the adelic $\Phi$-operator will map integral double cosets to nonintegral ones.

For a proof consider Krieg's extensive studies of the Hurwitz order in Kri87. Note that in the quaternionic case he writes $N(\delta)^{-k / 2}$ instead of $\tilde{N}(\delta)^{-k}$. All of his arguments are still valid, but we have to replace $p$ by $\#\left(\Lambda_{\mathfrak{p}} / \mathfrak{p} \Lambda_{\mathfrak{p}}\right)$. This does not change the underlying systems of linear relations for the images of the Hecke algebra's generators.

\section{EXAMPLES}

In this section we reproduce an example given in the unpublished dissertation Ens08, Remark 3.3.6]. Using our results it can now be completely understood. We also illustrate our result by two other examples.

Example 5.1 (An example found by Ensenbach). We deal with the hermitian case and $n=2$.

Ensenbach considered the following very concrete example. Let $\omega=\sqrt{-5}$ and $\Omega=\mathbb{Q}(\omega), K=\mathbb{Q}$. We set $M_{6}=[1,1+\omega]$ and $M=\left[M_{6}, 6 \imath(M)^{-\operatorname{tr}}\right]$. Then $M$ is an irreducible double coset with similitude 6 .

A more general construction is as follows. Consider two places $\mathfrak{p}, \mathfrak{q}$ which are inverse to each other in the class group of $\Lambda$. Choose primes $p$ and $q$ in $K$ above them. Let $m$ be a generator the ideal associated to $\mathfrak{p q}$. Then the double coset given the adelic matrix $\left[1, \ldots, 1, m, 1, \ldots, p q \imath(m)^{-1}\right]$ will generate a double coset contained in integral Hecke algebra. As an element of the integral Hecke algebra it is irreducible in the sense of Section 4 .

Example 5.2 (Abstract generators for the integral Hecke algebra). We want to illustrate the idea behind Lemma 4.1. Therefore, we will calculate the irreducible double cosets in the hermitian modular case with class number 2 for $n=2, K=\mathbb{Q}$.

So let us first consider those cosets which have prime similitude $p \in \mathbb{Q}$. If $p$ is inert the valuation of the elementary divisors of irreducible cosets are $(0,0,1,1),(0,1,1,2)$ 
and $(1,1,1,1)$. If $p$ ramifies, there are the irreducible double cosets $(1,1,1,1)$ and $(0,0,2,2)$. Moreover, if the ramified prime ideal above $p$ is principal, $(0,1,1,2)$ is irreducible; otherwise $(0,2,2,4)$ is irreducible.

If $p$ splits, we have to distinguish two cases. If it splits into principal ideals, all irreducible double cosets have similitude $p$, and the valuation of the local elementary divisors over $\Lambda_{\mathfrak{p}}$ are $(0,0,0,0),(0,0,0,1)(0,0,1,1),(0,1,1,1)$ and $(1,1,1,1)$. If it splits into nonprincipal ideals, those irreducible double cosets with similitude $p$ are $(0,0,0,0),(0,0,1,1)$ and $(1,1,1,1)$. In addition, there are three which have similitude $p^{2}$. They are $(0,1,1,2),(0,2,2,2)$ and $(0,0,0,2)$.

There remain those double cosets which have similitude $p_{1} p_{2}$ with distinct primes $p_{1}$ and $p_{2}$. They are the product of exactly two adelic double cosets $(0,1,1,2)$ if $p_{i}$ ramifies and $(0,1,1,1)$ or $(0,0,0,1)$ with similitude $p_{i}$ if $p_{i}$ splits.

Actually this is a minimal generating set with respect to inclusion of sets. This can easily be seen by the algebraic independence of the generators of the adelic Hecke algebra.

Example 5.3 (Concrete generators for the integral Hecke algebra). More concretely we can consider $\omega=\sqrt{-6}, \Omega=\mathbb{Q}(\omega)$ and $K=\mathbb{Q}$ as in the first example. Set $M_{2}=\left(\begin{array}{cc}\omega & -2 \\ 2 & \omega\end{array}\right)$. The component of the Hecke algebra above 2 is generated by $\left[M_{2}, 2 \imath\left(M_{2}\right)^{-\mathrm{tr}}\right],[1,1,2,2]$ and $[1,2,4,2]$. In analogy, the Hecke algebra above 3 is generated by $\left[M_{3}, 3 \imath\left(M_{3}\right)^{-\operatorname{tr}}\right],[1,1,3,3]$ and $[1,3,9,3]$. Here, we have $M_{3}=\left(\begin{array}{cc}\omega & -3 \\ 3 & \omega\end{array}\right)$.

In accordance with the first example the component above 6 is not the tensor product of the components above 2 and 3 . But we have to add one further generator $\left[M_{6}, 6 \imath\left(M_{6}\right)^{- \text {tr }}\right]$ with $M_{6}=\left(\begin{array}{cc}\omega & 2 \\ \omega & 3\end{array}\right)$.

\section{ACKNOWLEDGMENT}

The author thanks Aloys Krieg for helpful comments on the first version of this paper.

\section{REFERENCES}

[And69] Anatoli Andrianov, Rationality theorems for Hecke series and zeta functions of the groups $\mathrm{GL}_{n}$ and $\mathrm{Sp}_{n}$ over local fields, Izv. Akad. Nauk SSSR Ser. Mat. 33 (1969), 466-505. MR0262168 (41:6778)

[Ens08] Marc Ensenbach, Hecke-Algebren zu unimodularen und unitären Matrixgruppen über Dedekind-Ringen, Ph.D. thesis, RWTH Aachen University, 2008.

[HS83] Tatsuo Hina and Takashi Sugano, On the local Hecke series of some classical groups over p-adic fields, J. Math. Soc. Japan 35 (1983), no. 1, 133-152. MR679080|(84g:22027)

[Kri87] Aloys Krieg, The Hecke-algebras related to the unimodular and modular group over the Hurwitz order of integral quaternions, Proc. Indian Acad. Sci., Math. Sci. (Ramanujan Birth Centenary Volume) 97 (1987), no. 1-3, 201-229 (English). MR983615(90b:11048)

[Kri90] - Hecke algebras., Mem. Amer. Math. Soc. 435 (1990), 158 pp. (English). MR:1027069 (90m:16024)

[Rau10] Martin Raum, Elementary divisor theory for the modular group over quadratic field extensions and quaternion algebras, arXiv:0907.2762v2 [math.NT], submitted for publication, 2010

[Rei75] Irving Reiner, Maximal orders, Academic Press, London-New York-San Francisco, 1975, London Mathematical Society Monographs, No. 5. MR0393100 (52:13910)

[Shi59] Goro Shimura, Sur les intégrales attachées aux formes automorphes. (On integrals attached to automorphic forms), J. Math. Soc. Japan 11 (1959), 291-311 (French). MR0120372(22:11126) 
[Sie51] Carl Ludwig Siegel, Die Modulgruppe in einer einfachen involutorischen Algebra, Festschr. Akad. Wiss. Göttingen 1951, Math.-Phys. Kl., 157-167, 1951. MR0052460 (14:623b)

[Tam63] Tsuneo Tamagawa, On the $\zeta$-functions of a division algebra, Ann. of Math. (2) 77 (1963), 387-405. MR 0144928 (26:2468)

Lehrstuhl A für Mathematik, RWTH Aachen University, 52056 Aachen, Germany

E-mail address: Martin.Raum@matha.rwth-aachen.de

Current address: MPI für Mathematik, Vivatsgasse 7, 53111 Bonn, Germany

E-mail address: mraum@mpim-bonn.mpg.de 